\title{
Current Acupuncture Research on Opiate Drug Addiction
}

\author{
Jiu-Lin Wang ${ }^{1,2^{*}}$, Xiaozhen Fan², Marc Raedschelders ${ }^{1}$, GuojianHuang ${ }^{3}$, Fu-Lin Wang ${ }^{2}$ \\ ${ }^{1}$ Acupuncture Program, Grant MacEwan University, Edmonton, Canada \\ ${ }^{2}$ Bethune Oriental Medicine Center, Edmonton, Canada \\ ${ }^{3}$ Ankang Acupuncture Healing Centre Inc. St Mary's Rd, Winnipeg, Canada
}

"Correspondence author: Jiu-Lin Wang, Acupuncture Program, Grant MacEwan University, 9-302D, 10700-104 Ave NW Edmonton, AB, Canada. Tel: +1780497474; Fax: +17804974239; Email: wangj@macewan.ca

Citation: Wang JL, Fan X, Raedschelders M, Huang G, Wang FL (2018) Current Acupuncture Research on Opiate Drug Addiction. Curr Res Complement Altern Med: CRCAM-126. DOI: 10.29011/CRCAM-126/100026

Received Date: 02 February, 2018; Accepted Date: 08 February, 2018; Published Date: 16 February, 2018

\begin{abstract}
Opioid drug addiction is a worldwide social and medical problem. Acupuncture has emerged as the most evidence-based, non-pharmacologic treatment option that can be employed to decrease the public's opioid dependence. The present review systematically analyzed data from clinical trials and animal studies published in Chinese and English literature since 1970. Acupuncture point selection and combination, techniques, as well as possible mechanisms have been explored through the review. Acupuncture may play a more important role in the recovery stage by assisting to prevent relapse. Instead of replacing allopathic medications during opioid detoxification, acupuncture is more suitable to work as an adjuvant treatment to lower the dosage of pharmacological drugs and reduce the side effects from those drugs.
\end{abstract}

Keywords: Acupuncture; Effectiveness; Opioid drug addiction; Mechanism; TCM

\section{Introduction}

Drug addiction is a major worldwide social and medical problem. Opiates comprise one of the most commonly abused drugs [1,2]. Over 2.5 million Americans suffer from opioid use disorder which contributed to over 28,000 deaths resulting from overdose in 2014 [3,4] and the death toll doubled in 2016 [5]. Due to the severity of this epidemic, in 2011, the Obama administration released a white paper describing the administration's plan to deal with the opioid crisis. The administration's concerns about opioid addiction and accidental overdosing have been echoed by numerous other medical and governmental advisory groups around the world. On August 10, 2017, U.S. president Donald Trump declared the opioid crisis a national public health emergency, and August 31, 2017 was designated as "International Overdose Awareness Day" (IOAD) by the Centers for Disease Control and Prevention (CDC) [6].

Pharmacological treatment is still the main option for opioids drug addiction. These medications include methadone, buprenorphine, and naltrexone. But these medications are also potentially addictive [7].

To cope with the opioid crisis, various federal regulatory and oversightagencies, including the U.S. FoodandDrugAdministration (FDA), the National Academies of Sciences, Engineering, and Medicine (NASEM), and the Joint Commission have started to advise or mandate that healthcare systems and providers offer non-pharmacologic treatment options for pain control [8-10]. In this respect, acupuncture stands out as the most evidencebased and, readily available choice to fulfill this function [11].

\section{Acupuncture Treatment on Opioid Withdrawal Syndrome}

About 40 years ago, it was serendipitously observed that auricular acupuncture could relieve opiate withdrawal signs of addicts [12]. This finding received the attention of clinicians and research scientists around the world [13-15]. Different types of protocols have been developed since then. Song et al. [16] combined acupuncture with methadone maintenance treatment (MMT) to treat heroin addiction and found the results to be superior to MMT alone. 


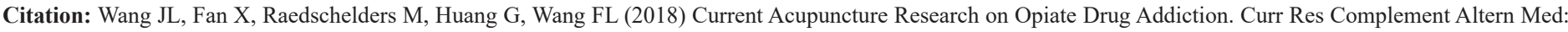
CRCAM-126. DOI: 10.29011/CRCAM-126/100026

Dr. Han's lab successfully relieved heroin withdrawal syndrome with single HANS's treatment. One pair of skin electrodes $(29 \times 29 \mathrm{~mm})$ was placed on Hegu point (LI-4) and the other at the palmer side on Laogong point (PC-8), to complete an electro-acupuncture(EA) circuit. Another pair of electrodes was placed on Neiguan (PC-6) and Waiguan (SJ-5) point to complete the EA circuit. These points are known to calm down the heart rate according to the theory of traditional Chinese medicine. A "densedisperse, DD" mode of stimulation was used, with a 2-Hz frequency alternated with $100-\mathrm{Hz}$ frequency, each lasting for 3 seconds. This mode of stimulation can release all four kinds of opioid peptides in the central nervous system (CNS) [17], producing a maximal opioidergic effect. The control group received the same treatment by placing the skin electrodes on the same sites, except that the electrodes were disconnected from the electric unit. One of the most prominent signs of withdrawal was tachycardia. The average heart rate of the patients in opioid withdrawal was 109 beats per minute (BPM) before treatment. The DD mode stimulation produced an immediate suppression of the tachycardia within 5-10 min, reaching $90 \mathrm{BPM}$ at the end of $30 \mathrm{~min}$. The effect is statistically very significant, even though it is short lasting, and return to its original level occurs in about $30 \mathrm{~min}$ [18-20].

A systematic literature review by Bake [21] indicate that the use of auricular acupuncture only with NADA protocol may not be effective in reducing acute opiate craving or withdrawal, it may be effectively utilized as an adjunctive treatment to increase treatment retention and decrease methadone detoxification and maintenance dosages in opioid use disorder.

Jin [22] successfully treated 32 cases of opioid drug addiction with MMT, together with auricular acupuncture, using the ear acupuncture points shenmen, lung, liver, heart, kidney, and spleen. Jiang successfully suppressed nausea symptoms from heroin addicts by needling PC6 with moderate stimulation [23].

\section{Acupuncture Treatment on Relapse Prevention}

It is well-known that drug addiction is a chronic, recurrent condition with high rate of relapse even after prolonged drug-free periods. According to statistics from different sources, the relapse rate can be as high as $95-99 \%$ [24]. Therefore, the final goal for treating drug addiction is not just detoxification (relieving or curing the withdrawal syndrome), but to relieve craving (psychic dependence) and to remove the compulsive behavior of drug seeking and taking after the detoxification. Current acupuncture study also demonstrated it could relieve anxiety, depression and sleep disturbance associated with opioid drug addiction.

Acupuncture treatments are thought to reduce the positive reinforcement properties of drugs by decreasing the amount of dopamine released when the drug is used. Without the surge of dopamine, the person's "high" or "buzz" is diminished, and he or she is less likely to use the drug in the future [3].

Craving is an important trigger of heroin relapse. Acupuncture was shown to suppress morphine self- administration in animals [25]. Lee et al. [26-29] demonstrated that acupuncture at Yanggu (SI-5) reduced morphine- seeking (craving) behavior following abstinence after both a fixed ratio (FR) schedule and progressive ratio (PR) schedule, with c-Fos expression in both VTA and NAc suppressed. Shenmen (HT-7) was also tested and showed attenuation of morphine self-administration at both low dose $(0.1 \mathrm{mg} / \mathrm{kg})$ and high dose on a FR schedule [30]. The active lever press decrease for FR schedule and breakpoint suppression for PR schedule were reversed by pretreatment with the selective GABAA antagonist bicuculline or the selective GABAB antagonist SCH50911, suggesting acupuncture on SI-5-mediated morphine craving through the GABA ergic pathway.

Human studies have been done by Dr. Han group as well. In a rehabilitation center, 171 former heroin addicts were recruited, all of whom had completed their detoxification process at least one month ago. The visual analog scale (VAS, $10 \mathrm{~cm}$ full scale) was employed to simulate the degree of craving. Subjects with a basal degree of craving lower than 2.0 were dismissed. The assessment was performed once a day for 30 consecutive days. Results obtained from the first 10 days and the last 10 days were taken as pre-control and post-control values, respectively, and those from the middle 10 days were taken to evaluate the effect of Transcutaneous Electrical Acupoint Stimulation (TEAS) on craving. The subjects were randomly divided into 4 groups, and 3 of them were given TEAS of 2,100 and $2 / 100 \mathrm{~Hz}$, respectively, for 30 min a day just after the measurement of craving. Another group was given mock TEAS, with barely sensible current at $15 \mathrm{~Hz}$ for 3 min, which was then switched off. The results showed that in all 4 groups, there was a general tendency of slow reduction of the craving. In the mock TEAS group, the slope remained stable during the whole period of observation. The $100 \mathrm{~Hz}$ group showed a small increase in slope in the TEAS treatment period without statistical significance. In contrast, the $2 \mathrm{~Hz}$ group and the $2 / 100 \mathrm{~Hz}$ group showed a sharp increase in slope, suggesting a significant lowering of the craving with considerable after effects. The conclusion is that for the suppression of craving, $2 \mathrm{~Hz}$ or $2 / 100 \mathrm{~Hz}$ TEAS was significantly more effective than $100 \mathrm{~Hz}$ TEAS $[31,32]$.

$\mathrm{Mu}$ et al. [33] evaluated craving clinically and performed a follow-up study to examine relapse rate. They compared the effects of acupuncture on bilateral Neiguan(PC6), Shenmen(HT7), Zusanli(ST36), Sanyinjiao(SP6) (Group 1) and that on bilateral Jiaji T5-7 and Shenshu(BL23) (Group 2). Needles were applied on these acupoints, whereas electro stimulation was done in the selected acupoints: ST36 and SP6 (Group 1), Jiaji T7 and BL23 (Group 2) with sparse wave on $5 \mathrm{~Hz}, 5 \mathrm{~mA}$ for 20 minutes, 3 times a week for 10 weeks; a sham group had electrodes fixed on ST36 


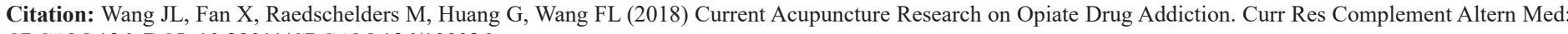
CRCAM-126. DOI: 10.29011/CRCAM-126/100026

and SP6 without actual stimulation, and a control group received no treatment. Craving recall (as measured by visual analogue scale) was lower in both acupuncture groups compared with the sham and control groups $(\mathrm{p}<0.01)$. Serum $\beta$-endorphin and dynorphin-A levels were also higher in the 2 acupuncture groups $(\mathrm{p}<0.01)$. Comparison of the 2 experimental groups revealed that EA on bilateral Jiaji T5-7 and BL23 seemed to alleviate heroin protracted withdrawal to a greater extent than bilateral PC6, HT7, ST36, SP6 ( $<<0.01)$. All groups were followed up 6 months after discharge. Of 120 cases, 98 were successfully contacted. The relapse rate in the Jiaji T5-7 and BL23 group was 77.3\%; that of PC6, HT7, ST36, and SP6 group was $88.5 \%$, whereas that of sham group was $90.5 \%$ and controls being $95.7 \%$. The relapse rate of the Jiaji T5-7 and BL23 group was significantly lower than the PC6, HT7, ST36, SP6 group, as well as the sham and control groups (p $<0.05$ ). It is known that the usual relapse rate within 6 months of detoxification is $>95 \%$, with protracted withdrawal symptoms a prominent factor in relapse [34].

Comparing to other traditional drug therapies, $\mathrm{Wu}$ et al. $[35,36]$ found acupuncture treatment significant suppress heroin's withdrawal syndrome after day 6 and suppress craving after day 8. Acupuncture can improve addicts' the memory loss and brain function.

Besides the craving, other psychological symptoms like depression, anxiety, sleep disturbance also contribute to relapse from opioid drug withdrawal $[37,38]$. Co- morbidity between substance abuse, depression, and anxiety is very common [39] and many studies take depression and anxiety as the outcome measures. Acupuncture has been scientifically proven to relieve depression [40,41]. A meta-analysis of acupuncture for opioid addictionassociated depression by Zhang et al. [42] included 2 trials that compared the effects of acupuncture with placebo acupuncture and with no treatment. Their results showed a statistically significant benefit of acupuncture in alleviation of depressive symptoms compared with both placebo acupuncture and no treatment. From the included trials, Hou et al. [43] randomly assigned 60 addicts who fulfilled the ICD-10 criteria to an acupuncture or control group. The acupuncture group showed significantly lower Hamilton Depression Rating Scale [44] than the control group. In the second trial from the analysis, $\mathrm{Mu}$ et al. [45] evaluated the effects on anxiety and depression by randomizing 120 heroin addicts who fulfilled the DSM-IV criteria to acupuncture on bilateral PC6, HT7, ST36, SP6 and LV-3 [Group 1], bilateral Jiaji T5,9,11 and BL23 (Group 2), sham electro stimulation group (Group 3), or control group (Group 4). For the 2 experimental EA groups, patient scores in the Zung Self-rating Depression Scale were lower than the sham and control groups $(p<0.05)$. Anxiety scores as indicated by the Zung Self-rating Anxiety Scale in the 2 EA groups were also lower than the sham and control groups ( $p$ $<0.01$ and $\mathrm{p}<0.05$ respectively). The effect of acupuncture on depression did not significantly differ between the 2 EA groups. Nonetheless for anxiety, acupuncture Group 2 (who received regular needling on bilateral Jiaji T9 and EA on Jiaji T5,11 and UB23 showed significant improvement compared with their Group 1 counterparts (i.e. regular needling on LV3 and EA on PC6, HT7, ST36, SP6) after 10 weeks of treatment.

$\mathrm{Xu}$ et al. [46] comparing T5 jia ji with PC6, found that combination of both has better effects on anxiety and depression caused by heroin addiction.

Sleep disturbance is another significant symptom in opioid withdrawal, positively correlated with relapse to heroin abuse. Zong et al. [47] compared opioid-associated sleep-related symptoms in 60 individuals. The experimental group was needled at bilateral PC6, HT7, ST36, SP6, Jiaji T5-7 and BL23, and electro stimulation was applied on ST36, SP6, Jiaji T7, and BL23 with continuous wave (3-5 Hz) for 20 minutes, 5 times a week for a total of 15 times. The control group received no treatment. Sleeping difficulties, such as difficulty falling asleep and easy awakening, could be alleviated by acupuncture with the above protocol $(\mathrm{p}<0.01)$. Zhu et al. [48] utilized the same acupuncture points as Zong et al. [46] but employed sparse wave EA ( $10 \mathrm{~Hz}, 5 \mathrm{~mA})$ for 20 minutes, 3 times a week for 10 weeks. They performed acupuncture at points on the back before moving on to acu-points on the limbs, and observed lowered scores for sleep-related symptoms $(p<0.001)$. TEAS has been shown to exert an immediate hypnotic effect in a third of heroin addicts [49]. Animal studies showed that during acute morphine withdrawal, rapid eye movement (REM) sleep, non-rapid eye movement (NREM) sleep, and total sleep time decreased, while sleep latency was prolonged. On the contrary, both $100-\mathrm{Hz}$ EA and 2-Hz EA significantly increased REM sleep, NERM sleep, and total sleep time [50]. These studies may aid in the design of treatment that improves sleep profile and thus reduces risk of relapse.

\section{TCM Etiopathology of Opioid Drug Addiction}

Opiates are acrid and bitter, warm and dry in nature. They move qi, warm the yang. Long term use of opiates will consume the qi and blood, damage body fluids, and further deplete the zang-fu organs and Yuan qi. After withdrawal of opiates, yang qi deficiency fails to warm the skin leading to chills, goose bumps; Wei qi deficiency fails to regulate skin pores leading to spontaneous sweats, night sweats; Endogenous cold congeals the collaterals leading to unbearable body ache. Deficiency of yin and blood fails to nourish the heart leading to palpitations, poor memory, insomnia, anxiety and irritability, fails to nourish the eyes leading to tearing, blurred vision, fails to nourish the face and pulse causing a pale complexion, a thin rapid pulse, fails to nourish the muscles and tendons resulting in muscle cramps; Spleen qi descends causing abdominal cramps, poor appetites, bloating, diarrhea; Stomach qi ascends leading to nausea, vomiting; Lung qi deficiency causes 


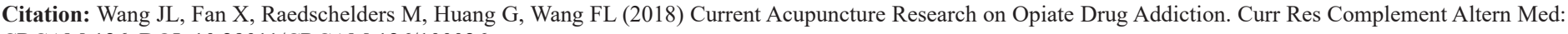
CRCAM-126. DOI: 10.29011/CRCAM-126/100026

cough, shortness of breath, nasal discharge; Kidney qi deficiency leads to low back pain, hairs loss, loose teeth, impotence, nocturnal emission etc.

Opioid drug withdrawal syndromes are usually time sensitive. Lung qi deficiency shows in the first three days, symptoms like shortness of breath, low voice, spontaneous sweats, chills, cold limbs, hyper sensitivity, nervousness etc present. From day 4 to 14, spleen qi deficiency shows up, including abdominal cramps and bloating, diarrhea, loose stools, craving, insomnia etc.

Dr. Gao [51] is of the opinion that opioid drug addiction results in "damage of qi, blood and body fluids", "Zang-fu organs damage" and "San Jiao damage"; Dr. Xie [52] holds that lung and spleen are the main organs involved in opioid drug addition, the chief pathology being deficiency and blood stasis; Dr. Song [53] thinks that the chief pathology is zheng qi deficiency, mixed with toxins and blood stasis, secondary with four patterns, i.e. , qi and blood deficiency, qi and yin deficiency, deficiency fire, yin and yang deficiency; Based on 107 cases of clinical observation, Dr. $\mathrm{Lu}$ [54] concludes that there is qi and yin deficiency mixed with turbid dampness and blood stasis in the early stage, qi and yin deficiency mixed with toxic heat and blood stasis in the middle stage, and yuan qi deficiency mixed with phlegm and blood stasis in the late stage. Dr. Bai [55] summarized opioid drug addiction into wind, phlegm, toxin, blood stasis, qi, and cold and deficiency seven pathological factors.

\section{Point Selection on Opioid Drug Addiction}

As shown in the systematic review by Zhang et al. [41] the acupoint Neiguan (PC-6) is the most frequently used point for management of psychological symptomsrelated to heroin addiction, followed by Zusanli (St36), Sanyinjiao(Sp6), Shenmen(Ht7), and Hegu (LI-4). These acupoints were also the most commonly used points in a systematic review by Lin et al. [56].

Cai [57] summarized the most frequently used points for heroin dependency are PC6, ST36, LI4, SP6, HT7, PC8, BL23, SJ5, DU20, GB34, Huatuojiaji, LI11, Sishencong(Figures 1-4).
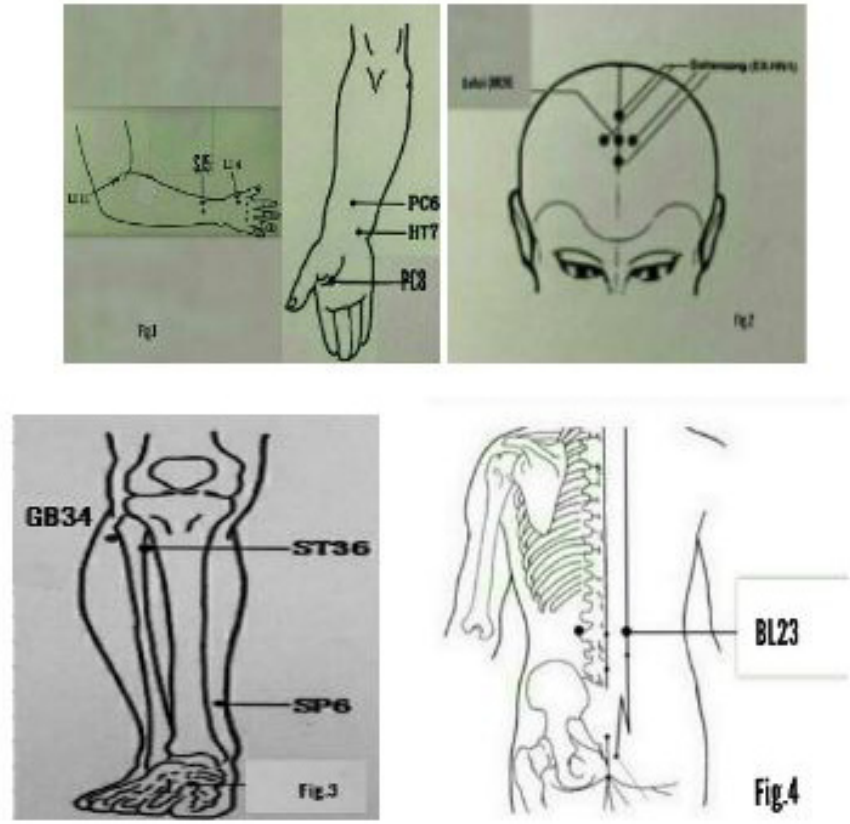

Figures 1-4: the most frequently used points for heroin dependency are PC6, ST36, LI4, SP6, HT7, PC8, BL23, SJ5, DU20, GB34, LI11, Sishencong.

Wang [58] using software like Excel, Weka to conclude ST36, SP6, PC6, LI4 and HT7 are top five points in acupuncture treatment on opioid drug addiction. Comparing to the top five points (HT7, DU20, SP6, PC6, BL23) in treatment of insomnia, there are $60 \%$ of similarity [59].

$\mathrm{Xu}$ et al. [45] collected 68 effective acupuncture formulae and 44 points from 66 clinical studies. The six most frequently used points were ST36, PC6, SP6, DU20, UB23 and HT7, of which ST36, PC6, and SP6 constituted a common point combination for acupuncture detoxification. The points for treatment of drug addiction are mainly from stomach, pericardium and spleen meridians, of which the points on lower limbs and head were the most commonly selected. The specific points in the treatment were five shu (stream) points, crossing points and lower He-Sea points. 
Citation: Wang JL, Fan X, Raedschelders M, Huang G, Wang FL (2018) Current Acupuncture Research on Opiate Drug Addiction. Curr Res Complement Altern Med: CRCAM-126. DOI: 10.29011/CRCAM-126/100026

\section{Acupuncture Techniques for Opioid Drug Addiction}

So far, auricular acupuncture, body acupuncture, scalp acupuncture, electro acupuncture, TEAS, laser acupuncture, moxibustion, imbedded needle and combined treatments have been reported for opioid drug addiction.

In early November 1972, Dr. Wen, a neurosurgeon in Hong Kong accidently used EA on body points, LI4, SI3, HT4 and TB9, together with two ear points (Brainstem, Shenmen) to relieve a patient's heroin addiction. In 1974, this protocol was further modified at the Lincoln Recovery Center in Bronx, New Yorkusing five ear points (Kidney, Liver, Lung, Shenmen, and Sympathetic (Figure 5) only. In 1985, the five-point auricular protocol was codified as the National Acupuncture Detoxification Association (NADA) protocol.

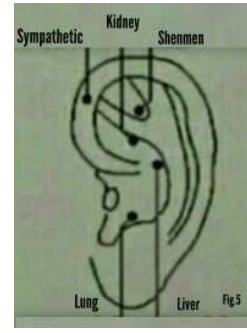

Figure 5: Five ear points (Kidney, Liver, Lung, Shenmen, and Sympathetic).

In 1965, Dr. Han of the department of Physiology, Beijing Medical University and his colleagues were involved in the study of the mechanisms underlying acupuncture anesthesia. With the discovery of endogenous opioid peptides activated in acupuncture anesthesia, it was evident for Dr. Han to study acupuncture treatment for drug addiction as well. Dr. Han applied EA on PC6, PC8, TE5, LI4 with different frequencies $(2 \mathrm{~Hz}, 100 \mathrm{~Hz})$ in several animal models and the human body. He demonstrated that acupuncture can suppress psychic dependence of opioid addiction. He further concluded that EA or HANS had the advantage over manual needling, TEAS was as effective as EA for the treatment of drug addiction. Comprehensive treatments like auricular acupuncture plus body acupuncture, body acupuncture plus MMT etc. are superior to treatments with only one method [24].

$\mathrm{Mu}$ et al. [33] concluded that electro acupuncture (EA) at Huatuojiaji (EX-B2) is better than at acupoints of the limbs with heroin dependency treatment.

\section{Acupuncture Mechanisms on Opioid Drug Addiction}

Acupuncture is commonly used for the treatment of pain. Mechanisms underlying acupuncture's analgesic effects have been extensively researched for over 60 years. In animal models, acupuncture and/or electroacupuncture has been shown to be effective for the alleviation of inflammatory, neuropathic, cancer related, and visceral pain. Mechano-transduction of the needling stimulus at specific points on the body triggers the release of ATP and adenosine, which bind to local afferents [60,61]. Ascending neural pathways involving $\mathrm{A} \beta, \mathrm{A} \delta$, and $\mathrm{C}$ sensory fibres have been mapped (using techniques such as single fiber recordings with Evans blue dye extravasations), as have been a mesolimbic analgesic loop in the brain and brainstem, descending pathway mechanisms, dopaminergic contributors, and cytokine, glutamate, nitric oxide, and gamma-amino butyric acid (GABA) effects. Acupuncture analgesia has been shown to involve several classes of opioid neuropeptides including enkephalins, endorphins, dynorphins, endomorphins, and nociceptin (also known as orphanin FQ). Among the non-opioid neuropeptides, substance P, vasoactive intestinal peptide, and calcitonin gene-related peptide have been investigated for their roles in both the analgesic and anti-inflammatory effects of acupuncture [62-65].

If acupuncture can accelerate the release of endorphins, it is natural to consider why not making use of acupuncture to relieve withdrawal syndrome of opiate addicts during drug abstinence. This thought was put into action in rats made dependent on morphine and in humans.

Early studies concluded that electrical stimulation at a low frequency $(2 \mathrm{~Hz})$ accelerates endorphin and encephalin production, whereas high-frequency stimulation (100 Hz) up-regulates dimorphic level. Later evidence suggested that $100-\mathrm{Hz}$ EA is effective in reducing withdrawal symptoms, with a suggestion that dimorphic suppresses withdrawal at the spinal level. The effect of $100-\mathrm{Hz}$ EA may be associated with BDNF activation at the VTA, while a cumulative effect of multiple $100-\mathrm{Hz}$ stimulation may be related to acceleration of dynorphin synthesis and down-regulation of p-CREB [66].

Yang's review provided clear evidence for the biological effects underlying the use of acupuncture to treat drug abuse [67]. Regarding positive reinforcement, acupuncture treatment activates GABAB receptors on the dopamine cell body and activates presynaptic $\kappa$-opioid receptors in the nucleus accumbens through dimorphic neurons, resulting in decreased dopamine release. Regarding negative reinforcement, acupuncture treatment stimulates encephalin neurons in the hypothalamus and interacts with $\mu$-opioid receptors to inhibit VTA GABAergic interneurons and thus increases dopamine release in the nucleus acumens.

Multiple animal studies [68-70] show that acupuncture can suppress NE, DA, 5-HT and increase $\beta$-EP, dynorphin-A in the central nervous system in morphine addicted rats. DA release is associated with drug withdrawal symptoms. Decrease of NE, 5-HT and increase of-EP, dynorphin-A are considered to benefit 


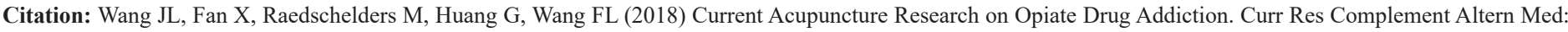
CRCAM-126. DOI: 10.29011/CRCAM-126/100026

anxiety and sleep disturbance. There are several animal models [71] that can be used to simulate the drug craving and relapse, conditioned place preference (CPP) is one of them. Wang et al. [72] first observed the effect of EA at 2, 100, or $2 / 100 \mathrm{~Hz}$ (DD) on morphine CPP expression in rats. The result showed that the CPP was significantly suppressed by EA with 2 and 2/100 Hz, but not of $100 \mathrm{~Hz}$. These results suggest that it is the low-frequency component of the EA mediated by $\mu$ and $\delta$ receptors that suppresses the CPP expression. Liang et al. [73] further localized the nucleus acumens (NAc) as the site of action. A single session of $2 \mathrm{~Hz}$ EA produced a significant increase of the release and the tissue content of encephalin in the NAc of morphine-induced CPP rats. This effect was stronger in rats that received 3 consecutive sessions of EA. These consecutive sessions of $2 \mathrm{~Hz}$ EA up regulated the mRNA level of preproenkephalin in the NAc of morphine-induced CPP rats. These findings support the feasibility of using $2 \mathrm{~Hz}$ EA for the treatment of opiate addiction.

Song et al. [74] observed heroin cue-induced changes of cerebral functional Magnetic Resonance Images (fMRI) as ab effect of acupuncture on ST36 in heroin-addicted volunteers. During heroin-visual stimulation, the markedly activated brain areas (increase of blood oxygen level-dependent signal) of fMRI were bilateral middle frontal gyrus, left cingulate gyrus, bilateral insula, occipital cortex, left cuneus, cerebellum, bilateral thalamus, right hippocampus and right amygdala etc. which are related to human's craving psychological activities. When acupuncture was provided, the heroin-addicted subjects showed apparent activation of the brain areas including the right anterior cingulate gyrus, right insula, thalamus, right hippocampal gyrus etc. and the activation degree was significant lower than in those with heroin related visual stimulation. He found acupuncture of ST36 can lessen heroin cue-induced activation degree of the brain areas involving psychological craving, suggesting that acupuncture can potentially suppress heroin craving in subjects with heroin addictions.

In the treatment for sleep disturbance, EA with both 100 $\mathrm{Hz}$ and $2 \mathrm{~Hz}$ significantly increased REM sleep, NERM sleep, and total sleep time. For craving, drug-seeking behavior was suppressed by acupuncture and was reversed by pretreatment with selective GABA antagonists, suggesting mediation of morphine craving through the GABAergic pathway. To observe the effect of electro acupuncture (EA) on the spatial learning-memory ability and the expression of NR 2 B subunit of NMDA receptor in the amygdale (AMG) in rats with morphine withdrawal, Sun [75] found both manual acupuncture and EA interventions can improve the learning-memory ability in morphine withdrawal rats, which is probably partially related to their effects in up-regulating the expression of NR $2 \mathrm{~B}$ in the AMG.

\section{Conclusion}

Acupuncture has been used to fight opioid drug addiction for over 40 years. Multiple protocols have been developed. EA and auricular acupuncture are two main techniques used in the current opioid drug addiction study. Existing evidence suggests that acupuncture as an adjuvant treatment may have the potential to decrease the necessary dosage of agonist and therefore alleviate the agonist's side-effects.

Acupuncture may have a detoxification effect in the acute stage but seems to play a more important role in the recovery stage by assisting to prevent relapse. Meta-analysis of clinical trials indicated the potential of acupuncture as a treatment for opioidassociated depression and anxiety, sleep disturbance and opioid craving. Animal studies demonstrated an influence of acupuncture on withdrawal symptoms. In the treatment of sleep disturbance, EA at both $100 \mathrm{~Hz}$ and $2 \mathrm{~Hz}$ significantly increased REM sleep, NERM sleep, and total sleep time. Craving and drug-seeking behavior was suppressed by acupuncture and was reversed by pretreatment with selective GABA antagonists, suggesting mediation of morphine craving through the GABA ergic pathway.

There is a clear evidence of acupuncture on opioid drug withdrawal in the animal studies. But the results from clinical studies are controversial. Most studies were hampered by their small study population, and lack of reporting of attrition rates and method of allocating patients. The studies also differed in their treatment regimen, type of waveforms if EA was the selected intervention, insertion depth (possibly due to different selected acupoints), and manipulation methods. This heterogeneity rendered it impossible to draw conclusions about the effectiveness of acupuncture as a treatment for opioid addiction. Other limitations that hindered the comprehensiveness of most existing studies include insufficient reporting of adverse events, practitioners' background, or response of patients. Commonly reported adverse events associated with acupuncture included needle pain (1-45\%), tiredness (2-41\%), and bleeding (0.03-38\%); fainting and syncope were common (86\%) [76]. Practitioners' background is important as it helps to ensure accurate representation of a clinical setting in the studies. Physician experience suggests that better therapeutic acupuncture effects are obtained by doctors with several years, or even decades, of clinical training ${ }^{55}$. Traditionally, the evocation of deqi(arrival of qi) response is often sought from patients but is not always reported in the studies. Deqi, the sensation of numbness and fullness at the site of stimulation, is believed to be important for acupuncture analgesia [77,78]. Scientific evidence showed that deqi was predictive of a positive outcome in osteoarthritis [79], and significant correlations were found of analgesia with numbness and soreness rating [80], suggesting that attributes of deqi sensation could be useful indicators of effective treatment.

There are some studies indicated no significant effect of acupuncture on opioid withdrawal symptoms [81-83]. All of these studies used auricular acupuncture instead of conventional body acupuncture. It is certainly worth investigating the synergistic 
Citation: Wang JL, Fan X, Raedschelders M, Huang G, Wang FL (2018) Current Acupuncture Research on Opiate Drug Addiction. Curr Res Complement Altern Med: CRCAM-126. DOI: 10.29011/CRCAM-126/100026

effects of a different combination of acupoints. Further studies of the presence and absence of needle manipulation, and type of stimulation would help practitioners to design appropriate and balanced treatment for opioid withdrawal symptoms.

Acupuncture demonstrated considerable potential as an adjuvant treatment. Regardless of the inconsistencies in therapeutic effect, key acupoints for opioid addiction have been identified. Considering the potential demonstrated by animal studies, further high-quality studies of specific acupoints and techniques that investigate the synergistic effects of a combination of acupoints may help to refine treatment protocol. The mechanism study will be shifted from single neuropeptide observation to the relationship between cognitive intervention and relapse.

\section{Acknowledgements}

I would like to thank all the participants in our research and the colleagues and research associates who collaborated on our studies that are included in this review. Special thanks go to Dr. Shuqing Tan for the charts provided in the review.

\section{Competing Interests}

The authors declare that they have no competing interests.

\section{References}

1. Liu ZM, Lv XX, Mu R (2002) A national survey on the basic situation of drug abuse in China. Chin J Prev Treat Drug Abuse 40: 27-30.

2. SAMSHA. Results from the 2007 National Survey on Drug Use and Health: National findings. Rockville, MD: Substance Abuse and Mental Health Services Administration, Office of Applied Studies. 2008.

3. Lin JG, Chan YY, Chen YH (2012) Acupuncture for the Treatment of Opiate Addiction. Evidence-Based Complementary and Alternative Medicine 2012: 739045

4. Bruce L (2011) Ear Acupuncture: A Tool for Recovery. Yale School of Medicine.

5. United States National Institute on Drug Abuse, National Institutes of Health. Overdose death rates. (2017-09) [2017-10-10].

6. Evans G (2017) On overdose awareness day, ninety people could die from opioids. (2017-08-31) [2017-10-10]. ahcmedia.com/blogs/2hicprevent/post/141375-today-isopioid-awareness-day-ninety-peoplewill-die.

7. Auerbach, Stuart (May 10, 1976). "Methadone Unable to End Heroin Use" (PDF). The New York Daily Press. p. 26. Retrieved 12 June 2012.

8. The Food and Drug Administration. Introduction for the FDA blueprint for prescriber education for extended release and long-acting opioid analgesics. (2017-05) [2017-08-19].

9. The National Academies of Sciences, Engineering, and Medicine. National strategy to reduce the opioid epidemic, an urgent public health priority, presented in new report. (2017-06-13) [2017-08-19].
10. Official Publication of Joint Commission Requirements (2017) new and revised standards related to pain assessment and management. Jt Comm Perspect 37: 3-4.

11. Fan Y, Miller D, Bolash B, Bauer M, McDonald J, et al. (2017) Acupuncture's Role in Solving the Opioid Epidemic: Evidence, Cost-Effectiveness, and Care Availability for Acupuncture as a Primary, NonPharmacologic Method for Pain Relief and Management-White Paper 2017. Journal of Integrative Medicine 15: 411-425.

12. Wen HL, Cheung SYC (1973) Treatment of drug addiction by acupuncture and 14 electrical stimulation. Asian J Med 9:138-141.

13. McLellan AT, Grossman DS, Blaine JD et al. (1993) Acupuncture treatment for drug abuse: a technical review. J Subst Abuse Treat 10: 569576.

14. Treatment Episode Data Set (TEDS) (2003) National Admissions to Substance Abuse Treatment Services. Rockville, MD: Department of Health and Human Services, Substance Abuse and Mental Health Services Administration, Office of Applied Studies: 1994-1999; Margolin. Acupuncture for Substance Abuse. Curr Psychiatry Rep 5: 333-339.

15. Attilio D'alberto (2004) Auricular acupuncture in the treatment of cocaine/crack abuse: a review of the efficacy, the use of the national acupuncture detoxification association protocol, and the selection of sham points. J Altern Complem Med 10: 985-1000.

16. Song XG, Zhang H, Wang ZH, Gu Y, Liu H (2005) Clinical observation on heroin withdrawal syndrome treated with combination of acupuncture and methadone. Chinese Acupuncture 22: 795-797.

17. J-S Han, Q Wang (1992) Mobilization of specific neuropeptides by peripheral stimulation of different frequencies. News PhysiolSci 7: 176180

18. Wu LZ, Cui CL, Han JS (1996) Effect of Han's acupoint nerve stimulator (HANS) on the heart rate of 75 inpatients during heroin withdrawal. Chin J Pain Med 2: 98-102.

19. Wu LZ, Cui CL, Han JS (1999) Treatment on heroin addicts by 4-channel Han's Acupoint Nerve Stimulator (HANS). J Beijing Med Univ 31: 239-242.

20. Wu LZ, Cui CL, Han JS (2001) Reduction of methadone dosage and relief of depression and anxiety by $2 / 100 \mathrm{~Hz}$ TENS for heroin detoxification. Chin J Drug Depend 10: 124-126.

21. Baker TE, Chang G (2016) the use of auricular acupuncture in opioid use disorder: A systematic literature review. Am J Addict 25: 592-602.

22. Jin T (2002) 32 cases of drug addicts treated with combination of auricular acupuncture and methadone. J. of Nanjing University of TCM 18: 302-303.

23. Jiang LB (2009) 78 cases of nausea caused by heroin dependency treated by needling PC6. Chin J Prevention and Treatment of Drug Abuse 15: 223

24. Han J, Cui C, Wu L (2011) Acupuncture-related techniques for the treatment of opiate addiction: a case of translational medicine. Front Med 5: $141-150$

25. Yoon SS, Kim H, Choi KH, Lee BH, Lee YK, et al. (2010) Acupuncture suppresses morphine self-administration through the GABA receptors. Brain Res Bull 81:625-630. 
Citation: Wang JL, Fan X, Raedschelders M, Huang G, Wang FL (2018) Current Acupuncture Research on Opiate Drug Addiction. Curr Res Complement Altern Med: CRCAM-126. DOI: 10.29011/CRCAM-126/100026

26. Lee BH, Ma JH, In S, Kim HY, Yoon SS, et al. (2012) Acupuncture at SI5 attenuates morphine seeking behavior after extinction. Neurosci Lett 529: 23-27.

27. Lee BH, Lim SC, Jeon HJ, Kim JS, Lee YK, et al. (2013) Acupuncture suppresses reinstatement of morphine-seeking behavior induced by a complex cue in rats. Neurosci Lett 548: 126-131.

28. Lee B, Shim I, Lee H, Yin CS, Park HK, et al. (2010) Morphine-induced locomotor response and Fos expression in rats are inhibited by acupuncture. Neurol Res 32: 107-110.

29. Bong Hyo Lee, Rong Jie Zhao, Byung Gwon Lee, Nam Jun Kim, Chae Ha Yang, et al. (2015) Acupuncture Suppresses Morphine Craving in Progressive Ratio Through the GABA System, Journal of Acupuncture and Meridian Studies 8: 175-182.

30. Lee BH, Ku JY, Zhao RJ, Kim HY, Yang CH, et al. (2014) Acupuncture at $\mathrm{HT7}$ suppresses morphine self-administration at high dose through GABA system. Neurosci Lett 576: 34-39.

31. Wang B, Zhang BG, Ge XC, Luo F, Han JS.(2003) Inhibition by peripheral electrical stimulation of the reinstatement of morphine-induced place preference in rats and drug craving in heroin addicts. J Peking Univ Health Sci (Beijing Da Xue Xue Bao Yi Xue Ban) 35(3): 241-247

32. Han JS, Trachtenberg Al, Lowinson JH.(2005) Acupuncture. In: Lowinson JH, Ruiz P, Millman RB, Langrod JG. Substance abuse: a comprehensive textbook. 4th ed. Chapter 49. Philadelphia: Lippincott, 743-782

33. Mu JP, Liu L, Fang W. Cheng JM, Zhao L (2010) Effect of electroacupuncture at Jiaji (EX-B 2) on drug craving of heroin addicts and $\beta$-EP and Dyn-A in plasma. Chinese Acupuncture 11: 881-885.

34. Jaffe JH. Drug dependence. In: Kaplan HI, Sadock BJ, editors. Comprehensive textbook of psychiatry. 4th ed. Baltimore: Williams \& Wilkins1985: 991.

35. Wu JM, Wei DX, Luo YF et al. (2003) Clinical study of acupuncture on detoxification and relapse prevention of heroin addicts. Academic Journal of Integrated Traditional Chinese and Western Medicine 1: 268-72.

36. Wei DX, Wu JM, Luo YF (2010) the effect of acupuncture on memory and brain functions of heroin addicts. Academic Journal of Chengdu University of TCM 33: 33-35.

37. Llorente del Pozo JM, Fernández Gómez C, Gutiérrez Fraile M, Vielva Pérez I (1998) Psychological and behavioural factors associated with relapse among heroin abusers treated in therapeutic communities. Addict Behav 23: 155-169.

38. Kaplan HB, Meyerowitz JH (1970) Social and psychological correlates of drug abuse. A comparison of addict and non-addict populations from the perspective of self-theory. Soc Sci Med 4: 203-225.

39. Merikangas KR, Kalaydjian A (2007) Magnitude and impact of comorbidity of mental disorders from epidemiologic surveys. Curr Opin Psychiatry 20: 353-358.

40. Dong B, Chen ZQ, Yin X, Li DT, Ma J, et al. (2017) The Efficacy of Acupuncture for Treating Depression-Related Insomnia Compared with a Control Group: A Systematic Review and Meta-Analysis. BioMed Research International 2: 1-11.

41. Schnyer R, Hitt S (2001) Acupuncture in the Treatment of Depression: A Manual for Practice and Research. Churchill Livingstone.
42. Zhang B, Chen Y, Cheng K, Shen X, Liu S.(2014) Efficacy of acupuncture for psychological symptoms associated with opioid addiction: a systematic review and meta-analysis. Evid Based Complement Alternat Med 2014: 313549.

43. Hou WG, Liang Y, Wang YX, Zong L, Chen YA (2009) Clinical observation of electro-acupuncture intervention on depression of heroin addicts after withdrawal. Chin J Drug Abuse Prev Treat 15: 11-13.

44. Hamilton M (1960) A rating scale for depression. J NeurolNeurosurg Psychiatry 23: 56-62.

45. Mu JP, Liu L, Cheng JM, Zhou LZ, AO JB, Wang J, et al. (2008) Clinical study of electroacupuncture treatment for post-withdrawal anxiety and depression in drug addicts. Shanghai J Acupunct Moxibustion 27: $1-3$.

46. Xu, et al. (2017) Research on the rule of selecting points for acupuncture treatment of drug addiction withdrawal syndrome based on data mining technology. Modern Journal of Integrated Traditional Chinese and Western Medicine Apr. 26 (11).

47. Zong L, Hou WG, Wang XX, An BW (2009) Clinical observation of acupuncture treatment on sleep disturbance with heroin addicts. Shanghai J. of Acupuncture. 4: 191-194.

48. Zhu ZC, Mu JP, Liang Y, Zong L, Hu J, Xu P. (2005) Clinical observation of electroacupuncture treatment on sleep disturbance with heroin addicts. Shanghai J of Acupuncture. 5: 6-8.

49. Wu LZ, Cui CL, Han JS (1995) Han's acupoint nerve stimulator for the treatment of opiate withdrawal syndrome. Chin J Pain Med 1: 30-38.

50. Li YJ, Zhong F, Yu P, Han JS, Cui CL(2011) Electroacupuncture treatment normalized sleep disturbance in morphine withdrawal rats. Evid Based Complement Alternat Med. 2011: 361054.

51. Gao XM, Song SL, Bai XJ (2001) scientific analysis on TCM treatment of drug addiction. Chinese $\mathrm{J}$. of prevention and treatment of drug abuse 9: 3-9.

52. Xie RQ (2012) TCM theory and differentiation on drug addiction. Chinese $\mathrm{J}$. of prevention and treatment of drug abuse 18: 327-329.

53. Song SL, Bai XJ (2003) TCM understanding and treatment on opioid drug addiction. Academical J. of TCM 18: 289-292.

54. Lu L, Liu YP, Yang BF (2008) TCM pattern analysis on 107 cases of drug addicts. Chinese $\mathrm{J}$. of prevention and treatment of drug abuse 14: $219-220$.

55. Bai XJ, Gao XM, Song SL (2006) The rule of TCM herbal use on drug addiction. J. of Traditional Chinese Herbal Medicine 31: 73-76.

56. Jaung-Geng Lin, Yuan-Yu Chan, Yi-Hung Chen (2012) Acupuncture for the treatment of opiate addiction. Evid Based Complement Alternat Med 2012: 739045

57. Cai S, Wu JM, Xu H (2008) the rule of acupuncture point selection on treatment of heroin dependency. Academical $\mathrm{J}$ of Chengdu Medical College 3: 170-172.

58. Wang J, Luo JX, Xu P (2012) Data analysis of point selection on treatment of opioid drug addiction. Academical J of TCM 30: 2060-2062.

59. Huang K, Liang S, Xu Y, Lu S (2015) Law of acupoint selection in acupuncture treatment for insomnia based on data mining method. $J$ of Chinese Acupuncture \& Moxibustion 35: 960-963. 
Citation: Wang JL, Fan X, Raedschelders M, Huang G, Wang FL (2018) Current Acupuncture Research on Opiate Drug Addiction. Curr Res Complement Altern Med: CRCAM-126. DOI: 10.29011/CRCAM-126/100026

60. Goldman N, Chen M, Fujita T, Xu Q, Peng W, et al. (2010) Adenosine A1 receptors mediate local anti-nociceptive effects of acupuncture. Nat Neurosci 13: 883-888.

61. Takano T, Chen X, Luo F, Fujita T, Ren Z, et al. (2012) Traditional acupuncture triggers a local increase in adenosine in human subjects. J Pain 13: 1215-1223.

62. Zhao ZQ (2008) Neural mechanism underlying acupuncture analgesia. Prog Neurobiol 85: 355-375.

63. Han JS (2011) Acupuncture analgesia: areas of consensus and controversy. Pain 152: S41-S48.

64. Han JS (2004) Acupuncture and endorphins. Neurosci Lett 361: 258261.

65. McDonald John L, Cripps Allan W, Smith Peter K (2015) Mediators, receptors, and signalling pathways in the anti-inflammatory and antihyperalgesic effects of acupuncture. Evid Based Complement Alternat Med 2015: 1-10.

66. Han JS, Zhang RL (1993) Suppression of morphine abstinence syndrome by body electroacupuncture of different frequencies in rats. Drug and Alcohol Dependence 31:169-175.

67. Yang $\mathrm{CH}$, Lee BH, Sohn SH (2008) A possible mechanism underlying the effectiveness of acupuncture in the treatment of drug addiction. Evidence-Based Complementary and Alternative Medicine 5: 257266.

68. Wu JM, Xie LL, Zhou QZ (2003) Acupuncture effects on DA, NE of anxiety mice model caused by morphine withdrawal. Sichuan J. of TCM 21: 18-19.

69. Cai XH, Shi FZ, Song XH (2011) Acupuncture effects on DA, 5-HT of rats with heroin dependency. Academical J. of Anhui TCM College 30: 38-41.

70. Zhou QZ, Cai DJ, Qiao XL (2006) Electroacupuncture effects on $\beta$-EP, $5-\mathrm{HT}, 5-\mathrm{HIAA}$ in hypothalamus of mice with morphine withdrawal anxiety. Academical J. of Chengdu TCM University 29: 43-45.

71. Markou A, Weiss F, Gold LH, Caine SB, Schulteis G, et al. (1993) Animal models of drug craving. Psychopharmacology (Berl) 112: 163-182.

72. Wang B, Luo F, Xia YQ, Hans JS (2000) Peripheral electric stimulation inhibits morphine induced place preference in rats. Neuroreport 11: 1017-1020.
73. Liang Y, Zong L, Li Y, Chen YL, Cheng B, et al. (2012) Therapeutic efficacy of acupuncture on post-withdrawal syndrome of heroin dependence. J Acupuncture Tuina Sci 10:155-159.

74. Song XG, Li XL, Wei KC, Li H, Zhao $Y$, et al. (2012) Influence of acupuncture on sleep disorders and anxiety in patients with heroin dependence. J Acupunct Tuina Sci 10:150-154.

75. Sun YZ, Liu TJ, Wei Z, Fan HY, Luan H (2015) Effect of electroacupuncture intervention on expression of NR2B subunit of NMDA receptor in amygdala during morphine withdrawal in rats. J. Acupuncture Study 40: 210-214.

76. Ernst E, White AR (2001) Prospective studies of the safety of acupuncture: a systematic review. Am J Med 110: 481-485.

77. Chapter 14, section II/3: Manipulations and arrival of Qi (needling reaction). In: Cheung XN, editor. Chinese acupuncture and moxibustion. Beijing: Foreign Language Press 1987: 325-327.

78. Stux G (1997) Technique of acupuncture. In: Stux G, Pomeranz B, editors. Basics of acupuncture. Berlin: Springer-Verlag 1997: 202-213.

79. Takeda W, Wessel J (1994) Acupuncture for the treatment of pain of osteoarthritic knees. Arthritis Care Res 7: 118-22.

80. Kong J, Fufa DT, Gerber AJ, Rosman IS, Vangel MG, et al. (2005) Psychophysical outcomes from a randomized pilot study of manual, electro, and sham acupuncture treatment on experimentally induced thermal pain. J Pain 6: 55-64.

81. Lua PL, Talib NS (2013) Auricular acupuncture for drug dependence: an open-label randomized investigation on clinical outcomes, healthrelated quality of life, and patient acceptability. Altern Ther Health Med 19: $28-42$.

82. Bearn J, Swami A, Stewart D, Atnas C, Giotto L, et al. (2009) Auricular acupuncture as an adjunct to opiate detoxification treatment: effects on withdrawal symptoms. J Subst Abuse Treat 36: 345-349.

83. Avants SK, Margolin A, Holford TR, KostenTR (2000) A randomized controlled trial of auricular acupuncture for cocaine dependence. Arch Intern Med 160: 2305-2312. 